\title{
Soundness Analysis of the Process of the Automatic Vending System Based on Petri Net
}

\author{
Lina Chen ${ }^{1, a}$, Xianwen Fang ${ }^{1, b^{*}}$ and Xiangwei Liu ${ }^{2, c}$ \\ ${ }^{1}$ School of Science,Anhui University of Science and Technology,Huainan 232001, China. \\ ${ }^{2}$ School of Management,Anhui University of Science and Technology,Huainan 232001, China.

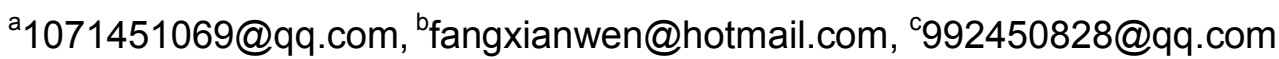

\begin{abstract}
Keywords: Petri net, data flow, control flow, soundness, automatic vending system Abstract: Automatic vending system is being widely used, the system of business process was mainly related to data flow and control flow, so the soundness analysis for business processes of automatic vending system is more difficult. The existing research about the soundness analysis of the process model is mainly from the control flow of the business process, and the influence of the data flow on the soundness of the business model is less considered. In this paper, based on the data flow and control flow of Petri net to analysis the soundness of automatic vending system process, which can not only analyzing the soundness of business process Petri net model from the control flow, but also further strengthened the influence of the data flow for the judgment on the soundness of the business process. Combining the example analysis shows that the method has certain feasibility.
\end{abstract}

\section{Introduction}

With the development of computer technology, the enterprises need to design and manage their business and information system, and to construct different workflow models to meet the different needs of business and customer service. So the correctness and soundness of the business model have become hot issues in the field of business process modeling. There are a lot of researches in this respect, but the main is the soundness of the business process model.

Usually, to design business processes based on an interactive approach. Firstly, to simulate control flow of business processes, including task sets which describe the behaviors. Then, expanding the control flow system with abstract data information, and finally generate an abstract business process model.Designing a work-flow model is a complicated and error-prone task even for experienced process designers. Therefore, cost-efficient design process, it is important that errors in the model are detected during the design phase rather than at run-time. Hence, in order to modify the program as soon as possible and adjusted to reduce costs, verification needs to be applied at an early stage.

In fact, data flow and control flow are two important components of the business process model, and provide important information for business process modeling, analysis and implementation, and have important constraints in model structure and behavior. According to literature [1], soundness can ensure that the nets would terminate and each task will not make the job nets deadlock or infinite loop, however, current technology to determine the soundness of business process is mostly limited in the control of flow field, without considering the data. Such as literature [2,3], since the study has some limitations, it might get the wrong conclusion that a business process model is unsound,in fact, it's sound after adding data.Many studies have subsequently started to pay attention to the data flow in the model role. In literature [4,5], the data flow and control flow had been applied to the process analysis and optimization,S. Moser in [6] clarify data-dependent clearly with BPEL-based business process , and find the data can be used to build more accurate dependence.Literature $[4,7]$ mentioned that data stream has influence on correctness and soundness, but didn't make a specific analysis.

\section{Motivating Example}

This part gives an example, through the elaboration of an example, analyzes the limitations of the existing methods in determining the soundness of the process model of the automatic vending system.

Fig. 1 shows a vending machine sales system,describing the process from select goods to coin 
payment.The graph includes two aspects:one is control flow, constraining the structure of business model, which makes the behavior of the service can be satisfied. For example,selecting the number of goods must be after selecting the types of goods, and only the customer put money,can the system distinguish money;only the customer meet the price needed,will the system send out correspond products automatically. The other is data flow, such as the type of the goods, the number of goods, and the prices, which are fixed data information and can transfer in the entire business process.

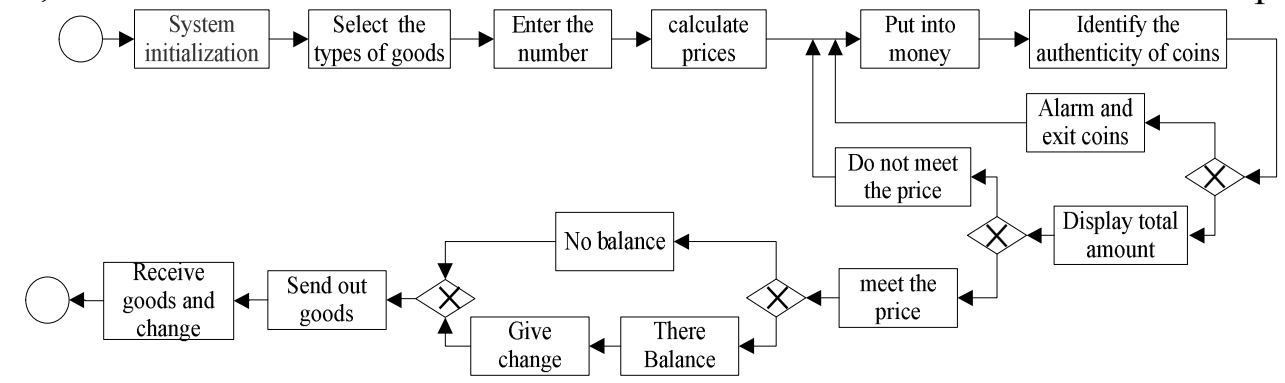

Fig. 1 a vending machine's shopping flow chart

From the above description, the business process model is normal operation without considering all the data in the figure, which meet the soundness requirements of the business process, but when take consideration the actual data information, for example the customer input is greater than the number of vending machine stock, the system is unable to provide the appropriate goods, thus making the system error, that is, the business process model can't work, and is unsound .Thus, to determine whether a business process model is sound or not we need to consider the control flow information, and the actual data information at the same time. In addition, we also found that, in order to serve our customers better with the actual ,business process model shown in Fig. 1 is needed to be improved, for example, by improving the business process model to avoid unsound situation.

\section{The analysis of business process Petri net under data constraints}

The soundness and the semantics of the business process Petri net under the data constraints

On the basis of $[8-11]$, we define the soundness of the business process as follows:

Definition 1 (soundness). If a business process model is sound, it satisfies the following conditions:

(1) $\forall m_{0} \rightarrow m_{n}, \exists \sigma$, st. $m_{n} \rightarrow m_{e}$, and $n=1,2,3, \cdots e-1$;

(2) $m_{e}=m\left(p_{e}\right)$;

(3) $\forall t, \exists m_{n}$ st. $m_{n} \stackrel{t}{\longrightarrow}$, and $m_{0} \rightarrow m_{n}, n=1,2,3, \cdots e-1$.

Literature [8] defines the nature of a data flow model about may be sound and must be sound . In general, the data flow model is sound and this ensures that all executable business processes are sound. If a data flow model can not sound, the corresponding executable data flow model is unsound. Therefore, in determining the soundness of the business process, it is not correct to only consider the control flow information, but should be combined with the specific situation and to extend control flow with abstract data information, and then to determine their soundness.

Dependency relations in business process Petri net with data constraints

The behavior profile of Petri net can be classified to discuss the relationship between the constraints, which is composed of sequential structure, exclusive structure, parallel structure and cross structure. The flowing gives the formal definitions about the behavior dependence and data dependence of the Petri net based on Petri net and the behavior profile .

Definition 2 (behavior dependency of the control flow Petri net). $P N_{C}=\left(P_{C}, T_{C}, F_{C}\right)$ is set up as a control flow Petri net of $P N=(P, T, F), \forall a, b \in T$, there are some relationships between $a$ and $b$ :

(1) direct dependence: $a \mapsto b$ i.e. $\left(a^{\bullet} \cap b \neq \phi\right) \wedge \exists \sigma=t_{1} t_{2}, \ldots, t_{n}, i \in\{1,2, \ldots, n-1\}: t_{i}=a \wedge t_{i+1}=b \vee$ $\left\{\exists M \in\left[P N_{C},[i]\right\rangle\right.$ st. $\left.\left(P N_{C}, M\right)[a\rangle \wedge\left(P N_{C}, M-\cdot a+a^{\bullet}\right)[b\rangle\right\}$; 
(2) indirect dependence: $a \propto b$

i.e. $\left(a^{\bullet} \cap^{\bullet} b \neq \phi\right) \wedge \exists c \in a^{\bullet} \cap^{\bullet} b \wedge \exists M \in\left[P N_{C},[i]\right\rangle$ st. $\left(P N_{C}, M\right)[a\rangle \wedge$

$\exists M^{\prime} \in\left[P N_{C}, M-^{\bullet} a+a^{\bullet}\right\rangle$ st. $\left(P N_{C}, M^{\prime}\right)[b\rangle \vee \exists \sigma=t_{1} t_{2}, \ldots, t_{n}, 1 \leq i<j<k, k \in\{3,4, \ldots, n\}:\left(t_{i}=a \wedge\right.$ $\left.t_{j}=c \wedge t_{k}=b\right)$;

(3) mutual dependence: $a \Leftrightarrow b$ i.e. $\{a \mapsto b \wedge b \mapsto a\} \vee\{a \propto b \wedge b \propto a\}$;

(4) mutual dependence: $a \| b$ i.e. $a \mapsto b \wedge b \mapsto \neg a \wedge a \neg \propto b \wedge b \neg \propto a$;

Control dependency relation is denoted as $C d e=\{\mapsto, \propto, \Leftrightarrow, \|\}$.

Four relationships described above indicate that the relationships between the activities are related to the premise of the activity.The relationships of the control flow Petri net mainly consider the relationships between the related activities. The behavior dependence of control flow is transitive.In the second part of the paper,the control flow Petri net dependency graph is presented in Fig. 2.

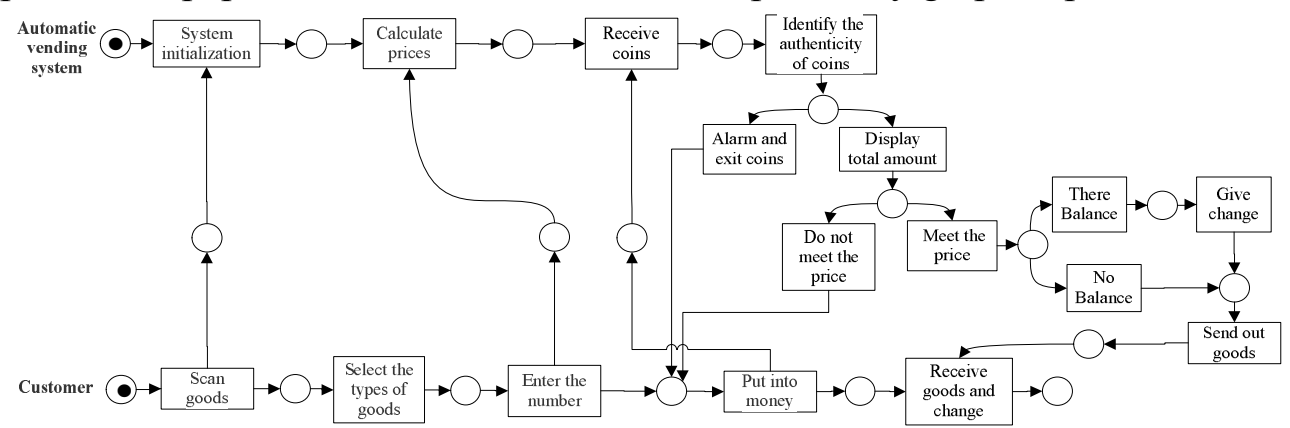

Fig. 2. The control flow Petri net dependency graph of auto sales system

Definition 3 (Data flow dependency). $P N_{D}=\left(P_{D}, T_{D}, F_{D}, W t, \mathrm{Re}\right)$ is defined as a data flow Petri nets of $P N=(P, T, F), \forall a, b \in T$, there are some relationships between $a$ and $b$ :

(1)The causal dependence: $a \leftrightarrow b$ i.e. $\left(a^{\bullet} \cap \bullet b \phi\right) \wedge \exists \sigma=t_{1} t_{2}, \ldots, t_{m}, i, j \in\{1,2, \ldots, m\}, i<j$ : $\left(t_{i}=a \wedge t_{j}=b\right) \vee a \notin T_{d}, \exists M \in\left[P N_{D}[i]\right.$, st. $\left(P N_{D}, M\right)[a\rangle \wedge\left(P N_{C}, M-\cdot a+a^{\bullet}\right)[b\rangle ;$

(2)The choice dependence $: a \triangleleft b$ i.e. $\exists \sigma_{1}=t_{1} t_{2}, \ldots, t_{m}, i, j \in\{1,2, \ldots, m\}, i<j:\left(t_{i}=a \wedge t_{j}=b \wedge c \notin \sigma\right)$ $\wedge a \leftrightarrow b \wedge a \leftrightarrow c$

(3)mutual independence: $a \| b$ i.e. $a \leftrightarrow b \wedge b \leftrightarrow a \wedge a \pitchfork b \wedge b \rtimes a$;

Data flow dependency relation is denoted $D d e=\{\leftrightarrow, \triangleleft, \|\}$.

Data flow dependency relationship in data flow Petri net shows that the generation of data information is related to the requirement of the activity. Data flow dependency relationship is transitive. The data flow dependence of the data flow Petri net, as mentioned, is shown in Fig. 3.

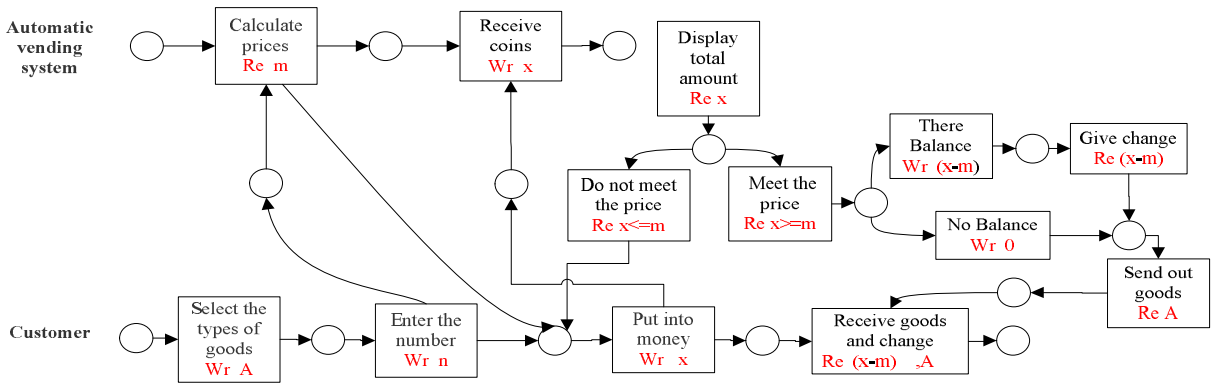

Fig. 3 data dependency graph of an auto sales system

\section{Algorithm:}

(1)Input the control flow Petri net $P N_{C}$ and the data flow Petri net $P N_{D}$;

(2) Run the control flow Petri net $P N_{C}$, if it runs normally, the business process model of control net is sound, then turn to the step (3), otherwise, the transitions which prevent the operation will be included in the set $W_{0}$; 
(3)Run the control flow Petri net $P N_{D}$, if it runs normally,both the control flow model and the data flow model of the business process model are sound, i.e.the business process model is sound, then exit,otherwise, the transitions which prevent the operation will be included in the set $W_{0}$, and turn to the step (4);

(4) Add the initial identification $M_{0}$, at the same time, lists all of the sequences through the region $W_{0}$ : $\sigma_{1}, \sigma_{2}, \sigma_{3}, \cdots, \sigma_{s}, 1 \leq i \leq s$, and then performing step (5),

(5) Take the sequence $\sigma_{i}$, for $\forall t_{i} \in W_{0} \cap \sigma_{i}$ and $t_{j} \in W_{0} \cap \sigma_{j}$, and $t_{i} \neq t_{j}, i<j$,

1) If ${ }^{\bullet} t_{i} \neq \phi$ or ${ }^{\bullet} t_{j} \neq \phi$, then $T_{1}=t_{i} \cup^{\bullet} t_{i} \cup t_{j} \cup^{\bullet} t_{j} \cup \cdots$, observe the presets in order and write down the enabled transitions and places until the end ; on the contrary $T_{1}=\sigma_{i}$;

2) If $t_{i}^{\bullet} \neq \phi$ or $t_{j}^{\bullet} \neq \phi$, then $T_{2}=t_{i} \cup t_{i} \cup t_{j} \cup t_{j} \cup \cdots$, observe the post-sets in order and write down the enabled transitions and places until the end ; on the contrary $T_{2}=\sigma_{i}$

3)Get the region: $W_{i}=T_{1} \cap T_{2}$;

(6)Back to the step (5) to obtain a sequence of operations for each execution sequence and perform the operation;

(7) Output the region which cause the problem in the business process model $W=\cup W_{i}$.

According to the above algorithm, analysis example, found that the instance model is unsound, and the transitions which make the model unsound are "select the types of goods", "enter the number" and "calculate prices", because when considering data information, since the customers do not know the number of existing goods in the automatic vending system, resulting that the number of goods that customer input is greater than the number of existing goods in the automatic vending system, which cause the system can not run normally, and can't meet the needs of customers. To solve this problem, just to improve the system so that the system displays the number of goods inventory in stock automatically when the customer choose the types of goods, so customers can choose at the prompt, and thus avoid the the occurrence of the above problems.

Considering the actual needs of e-commerce and the nature of Petri nets, get the improved vending process Petri net model, shown in Fig. 4 .

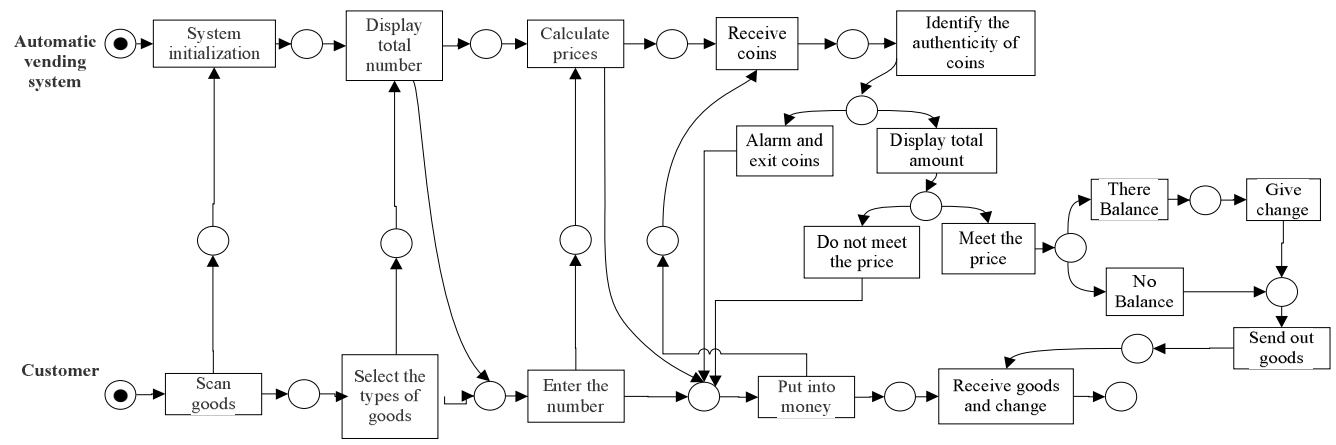

Fig. 4 sound automatic sales flow chart

\section{Conclusions}

With business process modeling approach to solve problems in the process model, since the constraints of practical application background, leading to the soundness verification of the established model of business process is very important. In this paper, based on the data flow and control flow of Petri net to analysis the soundness of automatic vending system process, which can not only analyzing the soundness of business process Petri net model from the control flow ,but also further strengthened the influence of the data flow for the judgment on the soundness of the business process. Combining the example analysis shows that the method has certain feasibility. The work of this paper makes up the shortages that only from the control flow to consider the structure constraint 
relationship of business process Petri net model, and has important reference value for the modeling of processes, analysis, implementation and verification.

In the future,we plan to use this method more widely on the detection of the soundness of service process model, and to detect the soundness of business process before runtime,so that we can make improvement and optimization timely.And hope to improve tools to detect the soundness of process model as soon as possible,which will help to integrate detecting and improving,so that realize the automation for business process modeling,combination and correlation analysis in the future.

\section{Acknowledgment}

We would like to thank the support of the National Natural Science Foundation of China under Grant No.61272153, No.61402011, No.61340003 and No.61170059, Anhui Provincial Natural Science Foundation (1508085MF111), Anhui Provincial Soft Science Foundation (12020503031), the Natural Science Foundation of Educational Government of Anhui Province of China (KJ2012A073, KJ2014A067), the youth academic backbone foundation of AUST, the Academic and Technology Leader Foundation of Anhui Province.

\section{References}

[1] W.M.P. Van der Aalst, The application of petri nets to workflow management, The J.Circuits, Syst. Comput. 8 (1) (1998)21-66

[2] N.Trcka,W.M.P.van der Aalst, N.Sidorova,Data-flow anti-patterns discovering data-flow errors in workflow, in:P.van Eck, J.Gordijin, R. Wieringa (Eds,),International Conference on Advanced Information Systems (CAiSE 2009), Lecture Notes in Computer Science,vol.5565,Springer-Verlag 2009,pp.425-439.

[3] N.Trcka,W.M.P.van der Aalst, N.Sidorova, Workflow completion patterns,IEEE International Conference on Automation Science and Engineering (CASE 2009),IEEE Press, 2009,pp.7-12.

[4] M. Batchelder and L. Hendren. Obfuscating java: the most pain for the least gain. In Proceedings of the 16th Internation Conference on Compiler Construction (CC'07), 2007.

[5] R. Vallee-Rai, E. Gagnon, L. Hendren, P. Lam, P. Pominville, and V. Sundaresan. Optimizing java bytecode using the soot framework: Is it feasible? In Proceedings of the 9th International Conference on Compiler Construction (CC' 00), 2000..

[6] S. Moser, A. Martens, K. Gorlach, W. Amme, and A. Godlinski. Advanced verification of distributed ws-bpel business processes incorporating cssa-based data flow analysis. In Proceedings of the IEEE International Conference on Services Computing (SCC’ 07), 2007.

[7] Filippo Bonchi, Antonio Brogi, Sara Corfini, and Fabio Gadducci. A Behavioural Congruence for Web Services[C]. International Symposilim on Fundamentals of Software Engineering, Lecture Notes in Computer Science .2007: (4767), 240-256.

[8] Natalia Sidorova, Christian Stahl, Nilkola Trcka,.Soundness verification for conceptual workflow nets with data:Early detection of errors with the most precision possible.Information Systems 36 (2011) 1026-1043.

[9] W. M. P. van der Aalst and M. Pesic. Decserflow: Towards a truly declarative service flow language. In Proceedings of International Conference on Web Services and Formal Methods (WS-FM'06), 2006.

[10] Zhangbing Zhou, Sami Bhiri, Manfred Hauswirth. Control and data dependencies in business processes based on semantic business activities. Integration and Web-based Applications \& Services. 2008: 257-263. 
[11] W.M.P. Van der Aalst ,K.M.van Hee, A.H.M. ter Hofstede,N.Sidorova,H.M.W. Verbeek,M. Voorhoeve, M.Wynn,Soundness of workflow nets:classification, decidability, and analysis, Formal Aspects Comput. 23 (3) (2011) 333-363.doi: 10.1007/s00165-010-0161-4. 